\section{Mortalidade infantil nos municípios brasileiros: uma proposta de método de estimação}

Wanessa da Silva de Almeida 1

Célia Landmann Szwarcwald 2

\section{Infant mortality rate in Brazilian municipalities: a proposal of an estimation method}

\footnotetext{
1,2 Laboratório de Informações em Saúde. Instituto de Comunicação, Informação Científica e Tecnológica em Saúde. Fundação Oswaldo Cruz. Av. Brasil, 4.365. Pavilhão Haity Moussatché. $2^{\circ}$ andar, Sala 225. Manguinhos. Rio de Janeiro, RJ, Brasil. CEP: 21.045-360. E-mail: wanessa.silva@icict.fiocruz.br
}

\section{Abstract}

Objectives: to propose a method to estimate the Infant Mortality Rate (IMR) by municipality, taking into consideration the underreporting of deaths and the IMR estimation in small population areas.

Methods: the method was developed on the basis of estimating the correction factors of death and live births by municipality, in the triennium 2009-2011, through indicators that characterize the completeness of vital information. A procedure to test if the corrected number of infant death achieved the expected minimum value was proposed accordingly to the municipality population size. In the case the correction is insufficient the predicted values of a multivariate regression were used to estimate the IMR.

Results: the estimation models of the vital information correction factors showed inverse and significant correlations with the completeness indicators. The predicted correction factors were applied to all municipalities located in the states with incomplete vital information. In only 230 municipalities, the correction factors were considered not sufficient.

Conclusions: the findings evidence that there are still great problems to surpass, such as the persistent inequalities related to socioeconomic development, access to health care, and omission of death reporting, which compromises the comprehension of a local situation.

Key words Infant mortality, Municipalities, Underregistration, Small-area analysis, Brazil

\section{Resumo}

Objetivos: propor um método para estimação do Coeficiente de Mortalidade Infantil (CMI) por municipio, levando em consideração a subenumeração de óbitos e a estimação do CMI em áreas de pequeno porte populacional.

Métodos: o método foi desenvolvido a partir da estimação de fatores de correção de óbitos e nascidos vivos por municipio, no triênio 2009-2011, através de indicadores construídos para caracterizar a cobertura das informações vitais. Foi proposto um procedimento para verificar se o número corrigido de óbitos infantis atingiu o valor mínimo esperado de acordo com o porte populacional do municipio. No caso de insuficiência do fator de correção, foram usados os valores preditos de uma regressão multivariada para estimar o CMI

Resultados: os modelos de estimação dos fatores de correção das estatísticas vitais mostraram correlações inversas e significativas com os indicadores que caracterizam a cobertura dos dados vitais. Os valores preditos dos fatores de correção foram aplicados para todos os municípios localizados nos estados que não têm informações vitais completas. Em apenas 230 municipios, a correção foi considerada insuficiente.

Conclusões: os achados evidenciam que existem, ainda, grandes problemas a superar, como as persistentes desigualdades relacionadas ao desenvolvimento socioeconômico, o acesso à assistência de saúde e à omissão da informação de óbito, que compromete o dimensionamento da situação local.

Palavras-chave Mortalidade infantil, Municipios, Sub-registro, Análise de pequenas áreas, Brasil 


\section{Introdução}

O crescente interesse na mensuração da mortalidade infantil, não só como indicador de saúde, mas também como um marcador do desenvolvimento humano, tem incentivado a sua estimação. Sua redução é, hoje, um dos objetivos das metas de desenvolvimento do milênio. ${ }^{1}$

O Coeficiente de Mortalidade Infantil (CMI) é calculado de forma direta pela razão entre o número de óbitos de menores de um ano e o total de nascidos vivos. No caso de países que não têm sistemas de informações de estatísticas vitais de registro contínuo ou cobertura precária dos dados de óbitos e nascidos vivos, o cálculo do CMI é problemático. ${ }^{2}$ Nesses países, o uso de inquéritos amostrais que coletam dados sobre todos os filhos nascidos vivos e mortos das mulheres pesquisadas tem sido frequente.3,4 Entretanto, devido às limitações dos métodos de mensuração indireta para a avaliação contínua e imediata da efetividade de ações e programas de saúde dirigidos às crianças menores de um ano de idade, o interesse pelas informações vitais de registro contínuo foi revigorado, tanto no plano nacional como no internacional.5,6

No Brasil, até a década de 1990, os métodos demográficos indiretos baseados em pesquisas domiciliares eram utilizados para estimar as probabilidades de morte por faixa de idade, especificamente, no primeiro ano de vida. 7 Entretanto, diante das restrições no uso de estimativas de mortalidade baseadas em pesquisas por amostragem,8,9 o Ministério da Saúde (MS) tem dirigido esforços à melhoria dos seus sistemas de informações vitais: o Sistema de Informações sobre Mortalidade (SIM) e o Sistema de Informações sobre Nascidos Vivos (Sinasc).

A divulgação das informações de mortalidade e nascidos vivos, por município, via internet, possibilitou detectar irregularidades locais. Foram propostos métodos para avaliação das informações vitais por município, utilizando-se indicadores construídos com dados dos próprios sistemas para avaliar sua cobertura e a regularidade.2,10 Outros procedimentos se fundamentaram no relacionamento de sistemas de informações. ${ }^{11,12}$ Além disso, o estabelecimento de metas relacionadas ao aumento da cobertura das informações de mortalidade, o desenvolvimento de painéis de monitoramento das informações, e a implantação de comitês de investigação do óbito fetal, infantil e materno, e óbitos mal definidos em todo o país, contribuíram para a melhora do registro dos óbitos. 13

Projetos de busca ativa, usados desde a década de 1980 para investigação dos problemas de captação dos eventos vitais 14 foram igualmente desenvolvidos. Foram realizadas várias pesquisas locais, percorrendo possíveis fontes de informações para encontrar óbitos e nascidos vivos não informados aos sistemas do MS.15-17 No ano de 2009, em iniciativa conjunta da Secretaria de Vigilância em Saúde do MS e a Fundação Oswaldo Cruz, foi conduzida uma pesquisa de busca ativa de óbitos e nascimentos em municípios da Amazônia Legal e do Nordeste, referente ao ano de 2008, com o objetivo de estimar fatores de correção das informações vitais por nível de adequação das informações. ${ }^{18}$

O método foi generalizado para a correção das informações de óbitos infantis e de nascidos vivos no período 2000-2009, por Unidade da Federação (UF) e Grande Região. ${ }^{19}$ Mostrou-se um aumento na cobertura de nascidos vivos de $92,5 \%$ a $95,9 \%$, e de óbitos infantis de $74,1 \%$ a $85 \%$, entre os anos 2000 e 2011, com ganhos expressivos nas regiões menos desenvolvidas do país.

Neste cenário de melhora na cobertura dos sistemas e valorização dos dados subnacionais, torna-se fundamental estimar a mortalidade infantil no nível de município. 8 O presente estudo tem como objetivo principal propor uma metodologia de estimação dos fatores de correção para as informações vitais e da mortalidade infantil por município brasileiro, contemplando a subenumeração de óbitos e a estimação do CMI em áreas de pequeno porte populacional.

\section{Métodos}

O método aqui proposto foi desenvolvido a partir da estimação de fatores de correção de óbitos e nascidos vivos (NV) por município brasileiro para o triênio 2009-2011. Devido à grande proporção (45\%) de municípios com menos de 10.000 habitantes, as análises foram realizadas por triênio para dar maior estabilidade aos indicadores.

Para todos os municípios de oito Unidades da Federação (Espírito Santo, Rio de Janeiro, São Paulo, Paraná, Santa Catarina, Rio Grande do Sul, Mato Grosso do Sul e Distrito Federal), os fatores de correção para óbitos (totais e infantis) e para NV foram estabelecidos como iguais a um, uma vez que as informações destas UF são consideradas completas, de acordo com critérios adotados pela Rede Interagencial de Informações para a Saúde (RIPSA). ${ }^{20}$

Para caracterizar o nível de cobertura das informações de mortalidade foi utilizado o Coeficiente Geral de Mortalidade Padronizado por idade 
(CGMP), tendo como padrão a população do Brasil para o ano de 2010. Conforme já apontado por outros autores, o CGMP pode ser utilizado para identificar falhas na cobertura das informações de registro de óbitos. $6,8,10$

Para um determinado município, o CGMP é calculado da seguinte forma:

$$
C G M P=\frac{\sum_{i}\left(\frac{O_{i}}{N_{i}} \times P_{i}\right)}{\sum_{i} P_{i}} \times 1000 ; i=0,1, \ldots, 10
$$

onde $\mathrm{O} i=$ número médio de óbitos no triênio na faixa de idade $i, \mathrm{~N} i=$ população do município no ano de 2010 na faixa de idade $i$ e $\mathrm{P}_{i}=$ população do Brasil no ano de 2010 na faixa de idade $i$. Foram consideradas as seguintes faixas etárias: $<1$ ano; $1-4$; $5-9 ; 10-14 ; 15-19 ; 20-29 ; 30-39 ; 40-49 ; 50-59 ; 60-$ 69 e 70 anos ou mais de idade, correspondentes a $i=0,1, \ldots, 10$, respectivamente.

Para caracterizar o nível de cobertura das informações de NV foi utilizada a razão entre nascidos vivos informados ao Sinasc e estimados (RZNV), onde o número esperado de NV é baseado na população menor de um ano. Para um determinado município, a RZNV é estimada por:

$$
R Z N V=\frac{N V_{i n f}}{N V_{e s p}}
$$

onde $\mathrm{NV}_{\text {inf }}=$ número informado de nascidos vivos do município e $\mathrm{NV}_{\text {esp }}=$ número esperado de nascidos vivos do município, dado por:

$$
N V_{e s p}=\frac{N_{\circ}}{0,989} \times 1,09
$$

No cálculo do número esperado de NV, o fator de 1,09 corresponde à correção da subenumeração da população em menores de um ano $\left(\mathrm{N}_{0}\right)$, enquanto o denominador corresponde à estimação do número de NV por meio da população de menores de um ano, considerando-se o CMI de 22 por $1000 \mathrm{NV}$ (valor aproximado do CMI das regiões Norte e Nordeste no ano de 2010). ${ }^{21}$

Para caracterizar o nível de cobertura dos óbitos infantis no nível municipal, foram adotados limites críticos inferiores, correspondentes à metade das estimativas do CMI por UF, no ano de 2010.

Para considerar na análise a probabilidade do evento ocorrer nos municípios de pequeno porte populacional, o critério foi estabelecido levando em conta a população do município. Primeiramente, em cada município, foi calculado o número mínimo esperado de óbitos infantis no triênio 2009-2011: onde $\mathrm{CMI}_{\min }$ é calculado pela metade da estimativa do CMI médio da UF no triênio, $\mathrm{NV}_{2009-2011}$ é o número médio de NV no triênio e a função TRUNC considera apenas a parte inteira do número decimal. Se o número informado de óbitos infantis for maior ou igual ao valor mínimo esperado $\left(\mathrm{O}_{0 \mathrm{~min}}\right)$, considera-se possível o número informado de óbitos infantis. Caso contrário considera-se que há subenumeração de óbitos infantis. É importante notar que em municípios pequenos, o número de óbitos infantis pode ser zero, e o CMI estar, portanto, abaixo do limite crítico inferior. Contudo, o número informado no triênio pode ser considerado possível em virtude do porte populacional do município.

Os fatores de correção para as estatísticas vitais por município brasileiro foram estimados com base nos resultados do estudo de Frias et al.,19 que propuseram um método simplificado para correção das estatísticas vitais por UF, na década de 2000 . Nesse estudo, foram gerados fatores de correção de óbitos totais e infantis, por UF e por categoria de CGMP, e de NV, por UF e categoria da RZNV, a partir dos eventos vitais encontrados na pesquisa de busca ativa de óbitos e nascimentos na Amazônia Legal e Região Nordeste e não informados aos sistemas de informações de estatísticas vitais do MS.22

Para a estimação dos fatores de correção de NV por município, nas demais UF, considerando-se a cobertura de $90 \%$ como satisfatória, calculou-se um indicador para medir falhas na cobertura de NV:

$$
R_{N V}=\frac{\min (R Z N V ; 0,9)}{0,9}
$$

Utilizou-se um modelo log-log tendo como variável dependente o logaritmo neperiano do fator de correção de NV, gerado no método simplificado de correção de NV por UF, 19 e como variáveis independentes o logaritmo neperiano da $R_{N V}$ e as variáveis binárias representativas dos efeitos das regiões geográficas (Amazônia Legal, Nordeste) e UF (Minas Gerais e Goiás).

Para a estimação dos fatores de correção para os óbitos infantis, os municípios foram agregados segundo região/UF (Amazônia Legal, Nordeste, Minas Gerais e Goiás) e categoria populacional $(<20.000, \quad 20.000-49.999, \quad 50.000-99.999$ e $100.000+)$. Em cada agregado, entre os municípios com CMI maior ou igual ao limite crítico inferior, foi calculado o CMI médio do agregado $\left(\mathrm{CMI}_{\mathrm{med}}\right)$.

Foram calculados dois indicadores para identificar falhas nas informações de óbitos: 


$$
R_{C G M P}=\frac{\min (C G M P ; 5,5)}{5,5} \quad e \quad R_{C M I}=\frac{\min \left(C M I ; C M I_{m e d}\right)}{C M I_{m e d}}
$$

O primeiro mede o afastamento do CGMP ao valor adequado de 5,5 por 1000 habitantes, enquanto o segundo mensura a distância do CMI do município ao CMI médio do agregado, entre os municípios com coeficientes superiores ao mínimo esperado.

Para a estimação dos fatores de correção de óbitos infantis por município, utilizou-se um modelo log-log, tendo como variável dependente o logaritmo neperiano do fator de correção de óbitos infantis por UF e categoria de CGMP gerado no método simplificado de correção das estatísticas vitais, 19 e como variáveis independentes o logaritmo neperiano da $\mathrm{R}_{\mathrm{CGMP}}$, o logaritmo neperiano da $\mathrm{R}_{\mathrm{CMI}}$ e as variáveis binárias representativas dos efeitos das regiões/UF.

No caso de insuficiência do fator de correção dos óbitos infantis, isto é, quando o número corrigido de óbitos infantis permaneceu menor do que o número mínimo esperado no triênio, estimou-se a mortalidade infantil através de um modelo multivariado.
$\mathrm{Na}$ análise multivariada, foram considerados todos os municípios com fatores de correção considerados como suficientes para estimar o CMI.

Para o modelo de regressão linear multivariada, utilizou-se a seguinte variável dependente (y):

$$
y=\operatorname{Ln}\left(\frac{C M I_{c r r}}{100-C M I_{c r r}}\right)
$$

onde Ln representa o logaritmo neperiano, $\mathrm{CMI}_{\mathrm{crr}}$ é o CMI corrigido pelos fatores de correção estimados por município. O uso desta função garante que y estará entre 0 e 100 por $1000 \mathrm{NV}$.

As variáveis independentes bem como a fonte da informação estão apresentadas na Tabela 1. Foram considerados indicadores socioeconômicos, demográficos, ambientais, de assistência em saúde e de adequação das informações de mortalidade.

Foi realizado um procedimento de seleção de variáveis passo a passo ("stepwise"), com critério de inclusão no nível de significância de $5 \%$ e de exclusão no nível de $10 \%$, utilizando-se o software de análise estatística SPSS. 23

\section{Tabela 1}

Indicadores utilizados no modelo de regressão multivariada segundo fonte e ano das informações.

\begin{tabular}{ll}
\hline & Socioeconômicos e demográficos \\
\hline Indicador & Fonte / Ano \\
\hline Renda domiciliar per capita & PNUD, 2010 \\
Índice de Gini & PNUD, 2010 \\
Proporção de domicílios com renda inferior a 1 salário mínimo & Censo demográfico, 2010 \\
Proporção de mães com menos de 8 anos de estudo & Sinasc, 2010 \\
Taxa de fecundidade total & PNUD, 2010 \\
Esperança de vida ao nascer & PNUD, 2010 \\
Proporção de população urbana & Censo demográfico, 2010
\end{tabular}

\section{Ambientais}

Indicador

Proporção de pessoas com abastecimento de água e esgotamento sanitário inadequados

Proporção de pessoas com serviço de coleta de lixo

Proporção de pessoas com fornecimento de energia elétrica
Fonte / Ano

Censo demográfico, 2010

Censo demográfico, 2010

Censo demográfico, 2010 
Indicadores utilizados no modelo de regressão multivariada segundo fonte e ano das informações.

\begin{tabular}{ll}
\hline \multicolumn{1}{c}{ Assistência à saúde } & Fonte / Ano \\
\hline Indicador & Sinasc, 2010 \\
\hline Proporção de mães com 7 ou mais consultas de pré-natal & SIM, 2010 \\
Proporção de óbitos com causa básica mal definida & CNES e Sinasc, 2010 \\
Número de leitos UTI neonatal por 1000 nascidos vivos & SIAB, 2010 \\
Cobertura do Programa de Saúde da Família (PSF) & Sinasc, 2010 \\
Deslocamento intermunicipal para o parto hospitalar (em km)28 & Fonte / Ano \\
Indicador & Adequação das informações de mortalidade \\
Logaritmo neperiano da $\mathrm{R}_{\text {CGMP }}$ & SIM, 2009-2011
\end{tabular}

PNUD= Programa das Nações Unidas para o Desenvolvimento; Sinasc= Sistema de Informações sobre Nascidos Vivos: $\mathrm{SIM}=$ Sistema de Informações sobre Mortalidade; CNES= Cadastro Nacional de Estabelecimentos de Saúde; SIAB= Sistema de Informação da Atenção Básica.

Após a seleção das variáveis conjuntamente associadas à variável resposta $\mathrm{y}$, o modelo foi utilizado para estimar o CMI entre os municípios onde o fator de correção foi considerado insuficiente.

Entre os municípios localizados nas oito UF que têm cobertura considerada completa das informações vitais, o $\mathrm{CMI}$ foi estimado pela razão entre o número informado de óbitos infantis e o número informado de NV. Entre os municípios localizados nas UF com cobertura incompleta das informações vitais com correção considerada suficiente, o CMI foi estimado pela razão entre o número corrigido de óbitos infantis e o número corrigido de NV. Entre os demais, o CMI foi estimado por:

$$
C M I=100 \times \frac{e^{y}}{1+e^{y}}
$$

Onde $\hat{y}$ é o valor predito da regressão multivariada.

Na Figura 1, apresenta-se um diagrama resumo do procedimento utilizado para estimação da mortalidade infantil para todos os municípios brasileiros.

A distribuição geográfica do CMI, estimado com os dados informados e após correção das informações vitais, para todos os municípios brasileiros, foi apresentada através de mapas do território nacional gerados no software TerraView. ${ }^{24}$ Para análise dos mapas, utilizou-se o percentil 90 do CMI calculado com as informações sem e após correção. 
Figura 1

Diagrama resumo do procedimento de correção e estimação da mortalidade infantil por município.

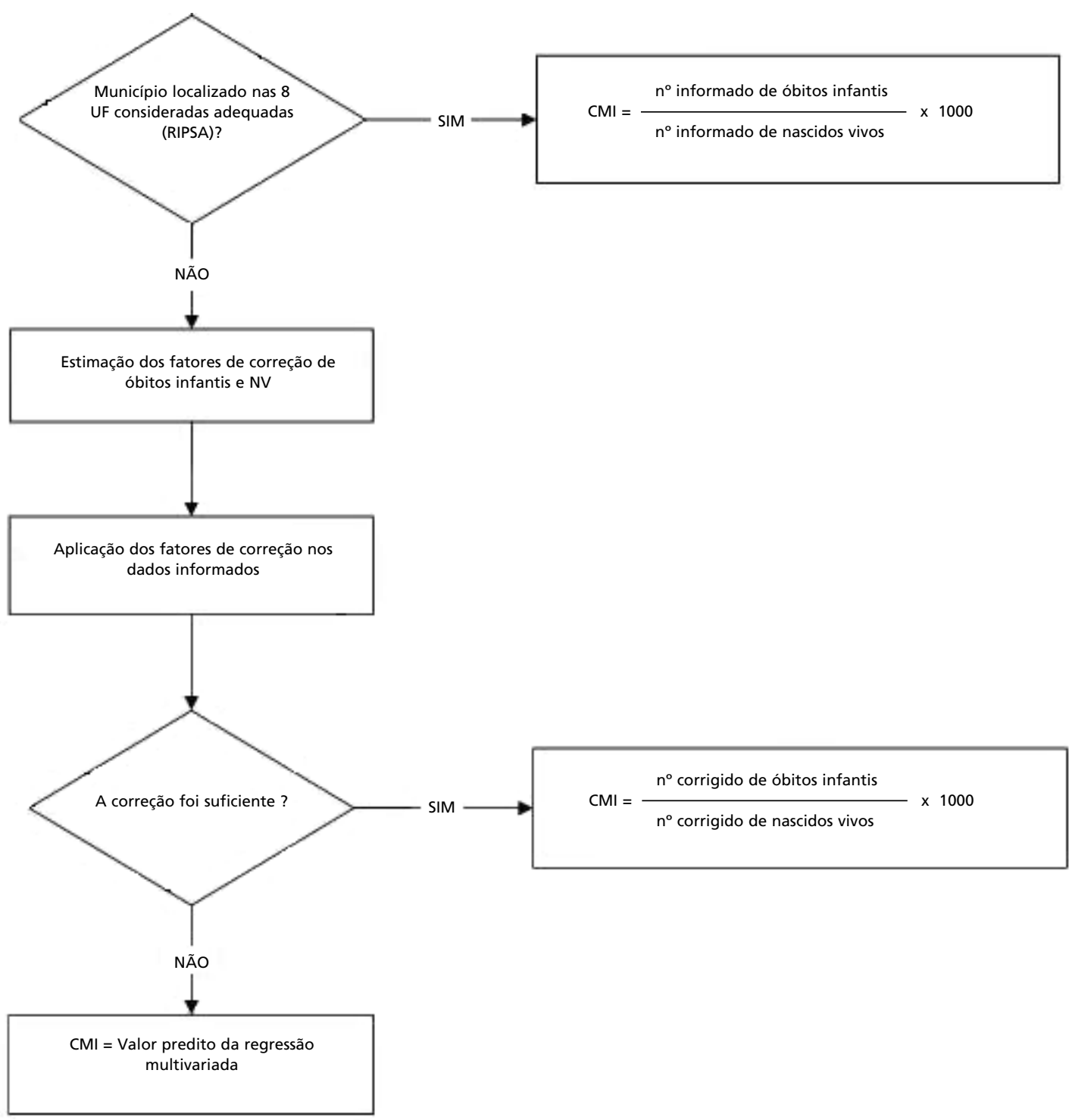




\section{Resultados}

Na Tabela 2, estão apresentados os resultados dos modelos utilizados para correção dos óbitos infantis e NV. Ambos os modelos mostraram elevados e significativos coeficientes de correlação múltipla ( $\mathrm{R}=0,935$ e $\mathrm{R}=0,870$, respectivamente).

O modelo utilizado para estimação dos fatores de correção para as informações de nascidos vivos mostra o efeito negativo da variável $\mathrm{R}_{\mathrm{NV}}$, ou seja, quanto mais o número informado de $\mathrm{NV}$ se aproxima do esperado, menor é a $\mathrm{R}_{\mathrm{NV}}$ e menores serão os fatores de correção. O mesmo efeito inverso foi encontrado no modelo de estimação dos fatores de correção para as informações de óbitos infantis: quanto mais o CGMP se distancia do valor adequado e o CMI informado do CMI médio do agregado, maiores serão os fatores de correção municipais (Tabela 2).

Utilizando-se os valores preditos dos fatores de correção de nascidos vivos e de óbitos infantis, as informações vitais foram corrigidas e aplicadas no cálculo do CMI para todos os municípios localizados nos estados que não têm informações vitais completas. Entretanto, em 230 municípios a correção não foi suficiente para que o número corrigido de óbitos infantis ultrapassasse o valor mínimo esperado no triênio.
Nos resultados do modelo multivariado verificou-se a relação inversa da proporção de pessoas com energia elétrica, abastecimento de água e esgotamento sanitário com os níveis de mortalidade infantil (Tabela 3). Igualmente, evidenciou-se a associação entre o CMI e o nível socioeconômico, expresso tanto pela proporção de pessoas com rendimento menor que um salário mínimo como pelo rendimento per capita. $\mathrm{O}$ índice de Gini foi positivamente associado ao CMI, isto é, quanto maior a desigualdade de renda, maior o CMI.

Quanto aos indicadores de assistência em saúde, o acesso geográfico ao parto hospitalar e a proporção de mães com sete ou mais consultas de pré-natal também mostraram-se preditores significativos do CMI: quanto maior o deslocamento intermunicipal para o parto, maior o CMI. Já o efeito do pré-natal foi inverso: maior a proporção de mães com número adequado de consultas, menor o CMI municipal. O percentual de óbitos mal definidos, reflexo, pelo menos, em parte da falta de assistência médica mostrou-se positivamente associado ao CMI (Tabela $3)$.

Em relação à adequação das informações de mortalidade, observou-se que nos municípios com pior nível de adequação das informações vitais, o CMI é mais alto (Tabela 3 ).

Tabela 2

Resultados dos modelos de regressão para estimação dos fatores de correção para as informações de nascidos vivos e óbitos infantis. Brasil, 2009-2011.

\begin{tabular}{|c|c|c|c|c|}
\hline Variável dependente & Coeficientes do modelo & $\boldsymbol{\beta}$ & $p$ & $\mathbf{R}$ \\
\hline Logaritmo neperiano do fator & (Constante) & $-0,021$ & $<0,001$ & 0,870 \\
\hline de correção para os nascidos & Logaritmo neperiano da $R_{N V}$ & $-0,672$ & $<0,001$ & \\
\hline \multirow[t]{4}{*}{ vivosa } & Região/UFb & & & \\
\hline & Amazônia Legal & 0,060 & $<0,001$ & \\
\hline & Nordeste & 0,043 & $<0,001$ & \\
\hline & Goiás & 0,057 & $<0,001$ & \\
\hline Logaritmo neperiano do fator & (Constante) & 0,142 & $<0,001$ & 0,935 \\
\hline de correção para os óbitos & Logaritmo neperiano da $\mathrm{R}_{\mathrm{CGMP}}$ & $-1,411$ & $<0,001$ & \\
\hline \multirow[t]{5}{*}{ infantisa } & Logaritmo neperiano da $\mathrm{R}_{\mathrm{CMI}}$ & $-0,043$ & $<0,001$ & \\
\hline & Região/UF b & & & \\
\hline & Amazônia Legal & 0,025 & $<0,001$ & \\
\hline & Nordeste & $-0,023$ & $<0,001$ & \\
\hline & Goiás & 0,084 & $<0,001$ & \\
\hline
\end{tabular}

a Gerados no estudo de Frias et al.19; b A categoria de referência utilizada foi o Estado de Minas Gerais. 
Tabela 3

Resultados do modelo de regressão multivariado utilizado para estimar o Coeficiente de Mortalidade Infantil (CMI) nos municípios onde o fator de correção foi insuficiente. Brasil, 2009-2011.

\begin{tabular}{lcc}
\hline Coeficientes do modelo c & $\boldsymbol{\beta}$ & $\boldsymbol{p}$ \\
\hline (Constante) & $-1,1412$ & $<0,001$ \\
Logaritmo neperiano da R CGMP & $-1,4456$ & $<, 001$ \\
Renda domiciliar per capita & $-0,0005$ & $<0,001$ \\
Proporção de pessoas com fornecimento de energia elétrica & $-0,0100$ & $<0,001$ \\
Proporção de mães com 7 ou mais consultas de pré-natal & $-0,0024$ & $<0,001$ \\
Proporção de domicílios com renda inferior a 1 salário mínimo & 0,0051 & $<0,001$ \\
Deslocamento intermunicipal para o parto hospitalar (em km) & 0,0003 & 0,002 \\
Índice de Gini & 0,5590 & $<0,001$ \\
Proporção de óbitos com causa básica mal definida & 0,0024 & 0,001 \\
Proporção de pessoas com abastecimento de água e esgotamento & 0,0026 & 0,002
\end{tabular}

c Variável dependente: logaritmo neperiano do CMI corrigido dos municípios onde o fator de correção foi suficiente.

O diagrama apresentado na Figura 1 mostra a síntese dos procedimentos para estimação da mortalidade infantil por município. Para os municípios que compõem estados considerados adequados segundo critérios da RIPSA, 20 o CMI foi calculado de forma direta para o triênio 2009-2011. Nos demais municípios, o CMI foi estimado pela razão entre o número corrigido de óbitos infantis e o número corrigido de NV. No caso de insuficiência da correção para atingir o número mínimo esperado de óbitos infantis de acordo com o porte populacional do município, foram utilizados os valores preditos do modelo multivariado (Tabela 3) para estimar o CMI.

A distribuição geográfica do CMI por município, com dados informados e corrigidos, apresentada na Figura 2, evidencia a subenumeração das informações de óbitos e nascimentos, principalmente nas
Regiões Norte e Nordeste do país. O percentil 90 do CMI informado para o Brasil foi de 24,5 por 1000 NV. Contudo, após correção, o percentil 90 aumentou para 32,2 por $1000 \mathrm{NV}$. Considerando o CMI calculado com os dados informados, $26,0 \%$ dos municípios apresentavam valores inferiores a 10 por $1000 \mathrm{NV}$. Esse percentual, entretanto, diminui para $11,6 \%$ quando se analisa o CMI estimado.

Percebem-se, ainda, as marcantes desigualdades do CMI estimado. Dos 5.565 municípios brasileiros, 250 apresentam CMI maior que 40 por $1000 \mathrm{NV}$, sendo que $40 \%$ são localizados na Região Nordeste. Por outro lado, nas Regiões Sul e Sudeste, mais do que $80 \%$ dos municípios têm CMI inferior a 20 por $1000 \mathrm{NV}$, enquanto nas regiões Norte e Nordeste, as proporções são $35 \%$ e $51 \%$, respectivamente. (Figura 2). 
Coeficiente de Mortalidade Infantil (CMI) por 1000 nascidos vivos, por município, utilizando dados vitais informados e corrigidos. Brasil 2009-2011.
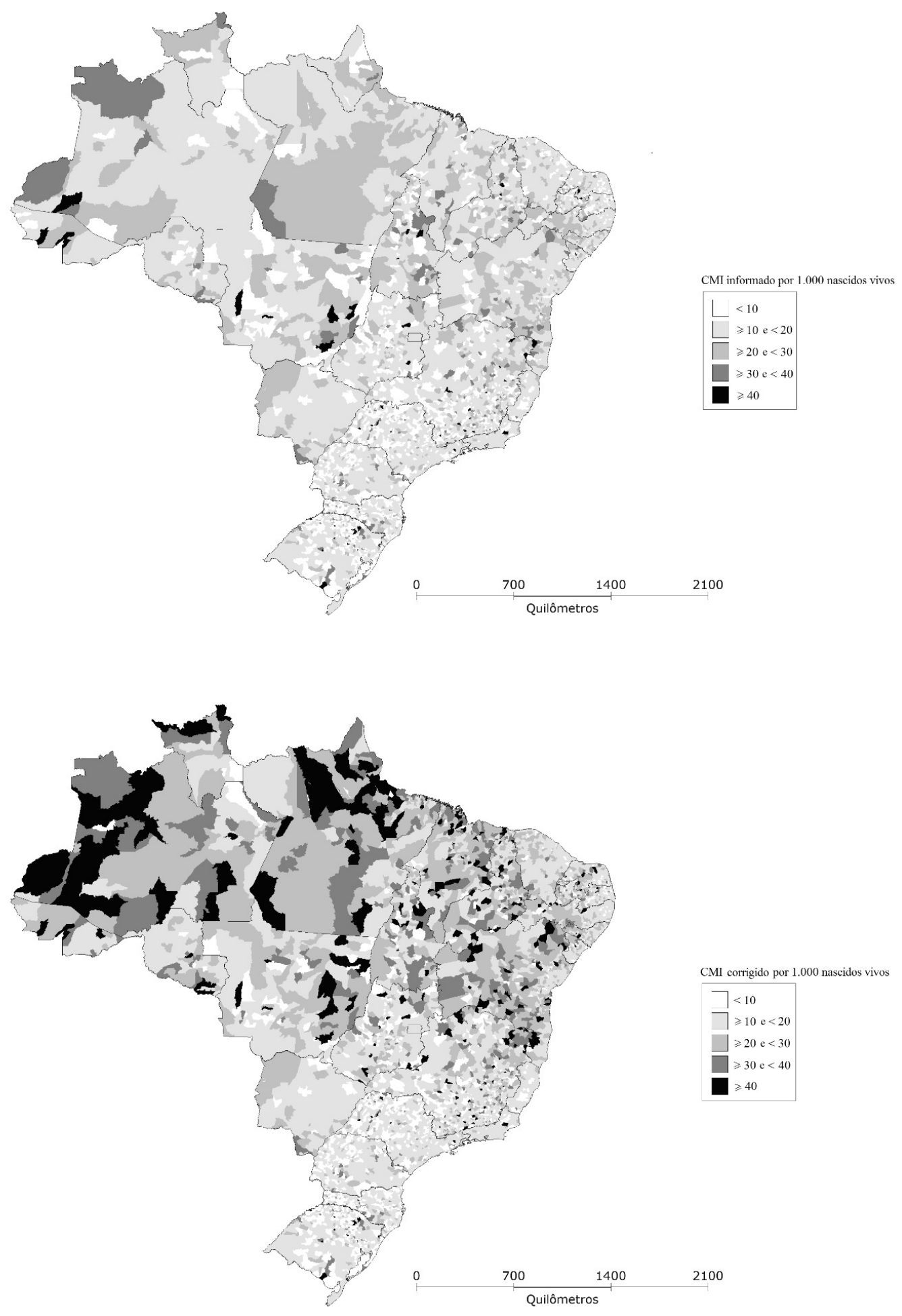


\section{Discussão}

Diante das restrições no uso de estimativas indiretas por município, já devidamente documentadas, as estimativas da mortalidade infantil pelo método direto têm ganhado importância crescente. 8,13,25

Com a consolidação do processo de municipalização, os sistemas de informações de eventos vitais ganharam destaque, particularmente por disporem de dados essenciais para o cálculo de indicadores de saúde constantes de diversos pactos nacionais e internacionais. ${ }^{13}$ Avançou-se em dispor, atualmente, de métodos de correção das estatísticas vitais por UF, a partir de procedimentos de busca ativa dos eventos vitais e avaliação da adequação das informações do SIM e do Sinasc. 10,18,19,22

De fato, até 2007 , apenas em oito UF, para as quais as informações vitais eram consideradas completas, o CMI era calculado pelo método direto com as informações do MS. Nas demais, as estimativas do CMI eram realizadas por métodos demográficos indiretos, utilizando as informações do Censo Demográfico, 2000 e das Pesquisas Nacionais por Amostra de Domicílio (PNAD).7,13 A partir de 2008, houve uma mudança de paradigma e o MS passou a divulgar o CMI calculado por meio das estatísticas vitais corrigidas em todas as UF.

Contudo, o método simplificado de correção das estatísticas vitais por UF, conforme proposto por Frias et al.19 e adotado pelo MS, não é diretamente aplicável aos municípios. Primeiro, porque a correção dos óbitos infantis é feita por faixas do CGMP, indicador da cobertura das informações de mortalidade. Assim, a dois municípios categorizados na mesma faixa de CGMP, um no limite inferior e outro no limite superior, corresponderia o mesmo fator de correção. Enquanto no nível de UF esse tipo de correção não é problemático, uma vez que se trata da estimativa do CMI médio dos vários municípios que compõem a UF, no nível de município, esse tipo de correção é inadequado.

Outro aspecto importante a ser levado em consideração na estimação do CMI por município é a flutuação das estimativas em áreas de pequeno porte populacional. Do total de municípios brasileiros, $70 \%$ têm menos de 20.000 habitantes e $23 \%$, menos de 5000 habitantes. É importante notar que os municípios pequenos são os que têm o pior nível de adequação das informações vitais. ${ }^{18}$

Para contornar esses problemas, o método sugere que as estimativas do CMI sejam feitas por triênio, que provê uma maior estabilidade. Além disso, oferece indicadores que caracterizam a cobertura das informações vitais por município brasileiro, que são fáceis de calcular e podem ser construídos por meio das informações dos próprios sistemas SIM e Sinasc. De maneira inovadora, o método propõe, adicionalmente, a análise de adequação do número informado de óbitos infantis, levando em conta a probabilidade de ocorrência do óbito infantil em municípios de pequeno porte populacional. Ou seja, a ausência de óbitos infantis em um determinado período não significa, necessariamente, CMI igual a zero, mas, simplesmente, que não houve chance do evento ocorrer. Já se o número informado de óbitos infantis for inferior ao mínimo esperado, significa que há problemas de subenumeração de óbitos.

Observa-se, ainda, que o procedimento estatístico utilizado permitiu realizar a estimação da mortalidade infantil nos municípios brasileiros, para o triênio 2009-2011, por meio de variáveis que mensuram o afastamento dos indicadores municipais a valores esperados se as informações fossem completas. No caso dos óbitos infantis, o fator de correção é calculado em função do afastamento do CMI informado ao CMI esperado, calculado entre municípios semelhantes da mesma UF e mesmo porte populacional com informações consideradas adequadas. Ou seja, quanto melhor o nível de adequação das informações, menor será o fator de correção estimado. Portanto, a mesma metodologia poderá ser aplicada para outros triênios, permitindo acompanhar a melhora das informações municipais de óbitos e nascimentos para o cálculo do CMI.

Em relação aos resultados obtidos após a correção dos dados vitais, mostrou-se grande iniquidade na mortalidade infantil. Enquanto no nível de estados, a redução mais expressiva do CMI nas Regiões Norte e Nordeste, na década de 2000, acarretou a diminuição das desigualdades regionais, 1,19 a disparidade do CMI persiste no nível de municípios: $4,2 \%$ dos municípios apresentam estimativas superiores a 40 por $1000 \mathrm{NV}, 24 \%$ destes localizados na Região Norte, e 40\% na Região Nordeste. Embora o Brasil venha avançando na redução da mortalidade infantil, os achados evidenciam que existem, ainda, grandes problemas a superar, como as persistentes e marcadas iniquidades relacionadas ao desenvolvimento socioeconômico, ao acesso à assistência de saúde, e à omissão da informação de óbito, que compromete o dimensionamento da situação local.

Os fatores de correção estimados para os óbitos infantis foram insuficientes em poucos municípios (230), caracterizados por terem porte populacional pequeno, com população média de 5000 habitantes, isto é, mesmo após a correção do número informado de óbitos infantis, a estimativa ficou abaixo do número mínimo esperado. Nesses municípios, diante 
da impossibilidade de uso das informações locais, o CMI foi estimado por modelos de regressão utilizando-se como variáveis independentes características socioeconômicas, demográficas e ambientais do município, de acesso e utilização de serviços de saúde e de adequação das informações de mortalidade.

Apesar do modelo de regressão multivariada ter sido utilizado apenas para estimar a mortalidade infantil onde a correção foi considerada insuficiente, os resultados foram bem interessantes e merecem discussão específica. Primeiramente, as associações do CMI com os indicadores de pobreza e de desigualdade de renda foram consistentes com a literatura. $26,27 \mathrm{Em}$ segundo lugar, é relevante destacar o efeito significativo da assistência em saúde, estabelecido pela realização de consultas prénatal, e pelo efeito do deslocamento intermunicipal para o parto hospitalar, que foi inversa e significativamente associado ao CMI, expressando as dificuldades de acesso geográfico ao parto hospitalar, sobretudo na região da Amazônia. 28 A proporção de óbitos mal definidos, que reflete, frequentemente, a falta de acesso à assistência médica, foi também inversamente associada ao CMI.16

É relevante apontar que no caso de insuficiência do fator de correção, a estimação do CMI pelos valores preditos do modelo de regressão multivariada é uma das possíveis alternativas metodoló- gicas. Frias et al. ${ }^{19}$ usaram o CMI médio dos municípios da mesma UF e mesmo nível de adequação das informações. Outra possibilidade é o uso métodos de análise estatística espacial. Além de serem utilizados como alternativa para superar a instabilidade das estimativas do CMI em pequenas áreas, se considerarmos a possibilidade de existência de autocorrelação espacial, com a variabilidade do CMI menor para áreas vizinhas, a inclusão da dimensão geográfica na análise pode trazer ganhos substanciais na interpretação e na detecção de padrões espaciais de variação da mortalidade infantil.29,30

Evidencia-se, entretanto, que embora as estimativas resultantes de modelos multivariados não substituam as estimativas feitas diretamente quando os dados vitais são adequados, servem, pelo menos, para mostrar que em determinados municípios o número informado de óbitos infantis está bem abaixo do esperado, de acordo com as suas condições socioeconômicas e de saúde. Por outro lado, investigações dos óbitos infantis e a ativação da busca de eventos vitais pelos serviços locais de saúde, precisam ser incentivadas nos municípios problemáticos. Esse tipo de análise possibilita evidenciar dificuldades contextuais relacionadas à mortalidade infantil que, sem dúvida, servirão para o aprimoramento de políticas e estratégias de intervenção no nível municipal.

\section{Referências}

1. Victora CG, Aquino EML, Leal MC, Monteiro CA, Barros FC, Szwarcwald CL. Saúde de mães e crianças no Brasil: progressos e desafios. Lancet. 2011; 377 (9780): 1863-76.

2. Szwarcwald CL, Andrade CLT, Souza Junior PRB. Estimação da mortalidade infantil no Brasil: o que dizem as informações sobre óbitos e nascimentos do Ministério da Saúde? Cad Saúde Pública. 2002; 18 (6): 1725-36.

3. Silva R. Child mortality estimation: consistency of underfive mortality rate estimates using full birth histories and summary birth histories. PLoS Med. 2012; 9(8): e1001296.

4. Korenromp EL, Arnold F, Williams BG, Nahlen BL, Snow RW. Monitoring trends in under-5 mortality rates through national birth history surveys. Int J Epidemiol. 2004; 33 (6): 1293-301

5. Viswanathan K, Becker S, Hansen PM, Kumar D, Kumar B, Niayesh H, Peters D, Burnham G. Infant and under-five mortality in Afghanistan: current estimates and limitations. Bull World Health Organ. 2010; 88 (8): 576-83.

6. Murray CJ, Rajaratnam JK, Marcus J, Laakso T, Lopez AD What can we conclude from death registration? Improved methods for evaluating completeness. PLoS Med. 2010; 7 (4): e1000262
7. Simões CC. Estimativas da mortalidade infantil por microrregiões e municípios. Brasília, DF: Ministério da Saúde; 1999.

8. Szwarcwald CL. Strategies for improving the monitoring of vital events in Brazil. Int J Epidemiol. 2008; 37 (4): 73844

9. Doctor HV. Variations in under-five mortality estimates in Nigeria: explanations and implications for program monitoring and evaluation. Matern Child Health J. 2013; 17 (8): $1355-8$.

10. Andrade CLT, Szwarcwald CL. Desigualdades sócio-espaciais da adequação das informações de nascimentos e óbitos do Ministério da Saúde, Brasil, 2000-2002. Cad Saúde Pública. 2007; 23 (5): 1207-16.

11. Rafael RAA, Ribeiro VS, Cavalcante MCV, Santos AM, Simões VMF. Relacionamento probabilístico: recuperação de informações de óbitos infantis e natimortos em localidade no Maranhão, Brasil. Cad Saúde Pública. 2011; 27 (7): 1371-9.

12. Mello-Jorge MHP, Gotlieb SLD. O Sistema de Informação de Atenção Básica como fonte de dados para os Sistemas de Informações sobre Mortalidade e sobre Nascidos Vivos. Inf Epidemiol SUS. 2000; 10 (1): 7-18. 
13. Frias PG, Szwarcwald CL, Lira PIC. Estimação da mortalidade infantil no contexto de descentralização do sistema único de saúde (SUS). Rev Bras Saúde Matern Infant. 2011; 11(4): 463-70.

14. Mello-Jorge MHP. Sub-registro dos eventos vitais: estratégias para a sua diminuição. Rev Saúde Pública. 1983; 17 (2): $148-51$

15. Façanha MC, Pinheiro AC, Fauth S, Lima AWDBC Silva VLP, Justino MWS, Costa EM. Busca ativa de óbitos em cemitérios da Região Metropolitana de Fortaleza, 1999 a 2000. Epidemiol Serv Saúde. 2003; 12 (3): 131-6.

16. Frias PG, Pereira PMH, Andrade CLT, Szwarcwald CL. Sistema de Informações sobre Mortalidade: estudo de caso em municípios com precariedade dos dados. Cad Saúde Pública. 2008; 24 (10): 2257-66.

17. Frias PG, Pereira PMH, Vidal AS, Lira PIC. Avaliação da cobertura do Sistema de Informações Sobre Nascidos Vivos e a contribuição das fontes potenciais de notificação do nascimento em dois Municípios de Pernambuco, Brasil. Epidemiol Serv Saúde. 2007; 16 (2): 93-101.

18. Brasil. Ministério da Saúde. Busca ativa de óbitos e nascimentos no Nordeste e na Amazônia Legal: Estimação da mortalidade infantil nos municípios brasileiros. In: Saúde Brasil 2010: uma análise da situação de saúde e de evidências selecionadas de impacto de ações de vigilância em saúde. Brasília, DF; 2011.

19. Frias PG, Szwarcwald CL, Souza Jr PRB, Almeida WS, Lira PIC. Correção de informações vitais: estimação da mortalidade infantil, Brasil, 2000-2009. Rev Saúde Pública. 2013; 47 (6): 1048-58.

20. RIPSA (Rede Interagencial de Informação para a Saúde). Indicadores básicos para a saúde no Brasil: conceitos e aplicações. 2 ed. Brasília, DF: Organização Pan-Americana da Saúde; 2008

21. Szwarcwald CL, Frias PG, Souza Júnior PRB, Almeida WS, Neto OL. Correction of vital statistics based on a proactive search of deaths and live births: evidence from a study of the North and Northeast regions of Brazil. Popul Health Metr. 2014; 12: 16

Recebido em 23 de março de 2014

Versão final apresentada em 25 de maio de 2014

Aprovado em 26 de junho de 2014
22. Brasil. Ministério da Saúde. Busca ativa de óbitos e nascimentos no Nordeste e na Amazônia Legal: Estimação das coberturas do SIM e do SINASC nos municípios brasileiros. In: Saúde Brasil 2010: Uma análise da situação de saúde e de evidências selecionadas de impacto de ações de vigilância em saúde. - Brasília: Ministério da Saúde; 2011 .

23. IBM SPSS Statistics for Windows, Version 21.0. IBM Corp. Released 2012. Armonk, NY: IBM Corp.

24. TerraView 4.2.2. São José dos Campos, SP: INPE, 2010. [acesso em 10 jan 2014]. Disponível em: www.dpi.inpe.br/ terraview.

25. Murray CJ, Laakso T, Shibuya K, Hill K, Lopez AD. Can we achieve Millennium Development Goal 4? New analysis of country trends and forecasts of under-5 mortality to 2015. Lancet. 2007; 370 (9592): 1040-54.

26. Szwarcwald CL, Souza Jr PRB, Damacena GN Consequences of inequality in the income distribution on the infant mortality inequalities. Int J Healthcare Insurance and Equity [periódico online]. 2013 [acesso em 13 jan 2014]. 1(2). Disponível em: http:///ijhie.com/IJHIE/ index.php/IJHIE/article/view/7/6.

27. Ram R. Income inequality, poverty, and population health: evidence from recent data for the United States. Soc Sci Med. 2005; 61 (12): 2568-76.

28. Almeida WS, Szwarcwald CL. Mortalidade infantil e acesso geográfico ao parto nos municípios brasileiros. Rev Saúde Pública. 2012; 46 (1): 68-76.

29. Asiimwe JB, Jehopio P, Atuhaire LK, Mbonye AK. Examining small area estimation techniques for public health intervention: lessons from application to under-5 mortality data in Uganda. J Public Health Policy. 2011; 32 (1): 1-14.

30. Ocaña-Riola R, Mayoral-Cortés JM. Spatio-temporal trends of mortality in small areas of Southern Spain. BMC Public Health. 2010; 10: 26.

\section{ERRATA}

No artigo "Mortalidade infantil nos municípios brasileiros: uma proposta de método de estimação" publicado no número 4 , volume 14, da Revista Brasileira de Saúde Materno Infantil, na página 333, onde se lê:

Primeiramente, em cada município, foi calculado o número mínimo esperado de óbitos infantis no triênio 2009-2011: onde $\mathrm{CMI}_{\min }$ é calculado...

Leia-se:

Primeiramente, em cada município, foi calculado o número mínimo esperado de óbitos infantis no triênio 2009-2011:

$$
O_{0 \min }=T R U N C\left(\frac{C M I_{\min } \times N V_{2009-2011}}{1000}\right)
$$

onde $\mathrm{CMI}_{\min }$ é calculado... 